\title{
Naringenin inhibits migration of bladder cancer cells through downregulation of AKT and MMP-2
}

\author{
ALEX CHIEN HWA LIAO ${ }^{1,2^{*}}$, CHIA-CHENG KUO ${ }^{3 *}$, YEI-CHUNG HUANG ${ }^{4}$, \\ CHI-WEI YEH ${ }^{4}$, YOU-CHENG HSEU ${ }^{5}$, JER-YUH LIU ${ }^{6,7}$ and LI-SUNG HSU ${ }^{4,8}$ \\ ${ }^{1}$ Division of Urology, Department of Surgery, Chi-Mei Foundation Medical Center; \\ ${ }^{2}$ Department of Senior Citizen Service Management, Chia Nan University of Pharmacy and Science, Tainan; \\ ${ }^{3}$ Department of Urology, Buddhist Tzu Chi General Hospital, Hualien; ${ }^{4}$ Institute of Biochemistry and Biotechnology, \\ Chung Shan Medical University; ${ }^{5}$ Department of Cosmeceutics, College of Pharmacy, China Medical University; \\ ${ }^{6}$ Center for Molecular Medicine, China Medical University Hospital; ${ }^{7}$ Graduate Institute of Cancer Biology, \\ College of Medicine, China Medical University; ${ }^{8}$ Clinical Laboratory, Chung Shan Medical University Hospital, \\ Taichung, Taiwan, R.O.C.
}

Received August 8, 2013; Accepted May 2, 2014

DOI: $10.3892 / \mathrm{mmr} .2014 .2375$

\begin{abstract}
Bladder cancer is one of the causes of cancer-related death and has a high mortality rate due to its metastatic ability. Naringenin, a bioactive compound predominantly found in citrus fruits, exhibits several cellular functions, including anti-oxidant, -lipidemia and -cancer abilities. However, the effects of naringenin on bladder cancer cells are yet to be elucidated. The present study investigated the molecular mechanisms underlying the effects of naringenin on the migration of TSGH-8301 bladder cancer cells. Treatment with naringenin at doses ranging between 0 and $300 \mu \mathrm{M}$ over a period of $24 \mathrm{~h}$ was found to reduce cell viability. Furthermore, zymography and western blot analysis revealed that naringenin reduced the expression of matrix metalloproteinase (MMP)-2 in a dose-dependent manner, and repressed its activity. Naringenin also reduced TSGH-8301 cell migration in a concentration-dependent manner, as evidenced by wound healing and Transwell ${ }^{\circledR}$ assays. In addition, naringenin was found to inhibit AKT activity and block the nuclear translocation of nuclear
\end{abstract}

Correspondence to: Professor Jer-Yuh Liu, Graduate Institute of Cancer Biology, College of Medicine, China Medical University, 6 Hsueh-Shih Road, Taichung 40402, Taiwan, R.O.C.

E-mail: jyl@mail.cmu.edu.tw

Professor Li-Sung Hsu, Institute of Biochemistry and Biotechnology, Chung Shan Medical University, No. 110, Section 1, Jianguo North Road, Taichung 40201, Taiwan, R.O.C.

E-mail:1shsu405@yahoo.com.tw

*Contributed equally

Key words: bladder cancer, migration, matrix metalloproteinase-2, naringenin factor $\kappa$-light-chain-enhancer of activated B cells. In conclusion, the findings of the present study show that naringenin is capable of inhibiting bladder cancer cell migration through the downregulation of the AKT and MMP-2 pathways.

\section{Introduction}

Flavonoids, extracted from traditional Chinese herbs, vegetables and fruit, have attracted much attention due to their antitumor activities. Several flavonoids have been shown to inhibit the proliferation and migration of bladder cancer cells, including curcumin, which has been found to induce apoptosis and repress bladder tumor growth in vitro and in vivo (1), and myricetin, which has been shown to arrest T24 bladder cancer cells at $\mathrm{G}_{2} / \mathrm{M}$ through the downregulation of cyclin B1 and the cyclin-dependent kinase cell division control protein 2 homolog (2). Myricetin also suppresses the migration of bladder cancer cells by decreasing matrix metalloproteinase (MMP)-9 expression (2). Butein has been reported to inhibit the migration and invasion of bladder cancer cells by reducing the expression and activity of extracellular signal-regulated kinase (ERK) 1/2 (3), and epigallocatechin-3-gallate (EGCG) has been shown to induce apoptosis in TSGH-8301 cells through the suppression of AKT activity and the modulation of heat shock protein 27 expression (4). Furthermore, EGCG has been observed to reduce AKT activity, deactivate nuclear factor $\kappa$-light-chain-enhancer of activated B cells $(\mathrm{NF}-\kappa \mathrm{B})$ and downregulate MMP-9 expression, consequently reducing the migration and invasion of T24 bladder cancer cells (5).

Naringenin, a bioactive flavonoid enriched in grapefruits and citrus fruits, exhibits a wide range of pharmacological activities. Frydoonfar et al (6) demonstrated that naringenin significantly inhibited proliferation in HT29 colon cancer cells. Naringenin treatment also decreased viability and lung metastasis in B16-F10 melanoma cells, with metastasis reduced by $63 \%$ compared with the non-treated groups (7). Furthermore, naringenin has been found to inhibit $\mathrm{N}$-nitrosodiethylamine 
(NDEA)-induced hepatocarcinomas in rats (8) and to exhibit anti-migration effects. Naringenin inhibits the tumor necrosis factor- $\alpha$-induced migration of smooth vascular muscle cells by elevating heme oxygenase-1 expression (9). Recently, Lou et al (10) demonstrated that naringenin repressed pancreatic cancer cell migration and invasion through the downregulation of transforming growth factor- $\beta$-induced epithelial to mesenchymal transition markers, including vimentin, N-cadherin, MMP-2 and MMP-9.

Bladder cancer is one of the most common malignant neoplasms worldwide and is associated with a high mortality rate among urological neoplasms (11). Emerging research has indicated that extracts from traditional herbs exhibit beneficial effects for the treatment of bladder cancer, including pro-apoptotic and anti-migratory effects. However, the effects of naringenin on bladder cancer cells are yet to be elucidated. The present study investigated the molecular mechanisms underlying the effect of naringenin on the migration of TSGH-8301 bladder cancer cells.

\section{Materials and methods}

Cell culture. Human bladder carcinoma TSGH-8301 cells (Bioresources Collection and Research Center, Food Industry Research and Development Institute, Hsinchu, Taiwan) were maintained in RPMI-1640 medium supplemented with $10 \%$ fetal bovine serum (FBS), $100 \mathrm{U} / \mathrm{ml}$ penicillin and $100 \mu \mathrm{g} / \mathrm{ml}$ streptomycin. Cells were incubated at $37^{\circ} \mathrm{C}$ in a humidified atmosphere with $5 \% \mathrm{CO}_{2}$.

MTT assay. Cells were seeded on 24-well plates at a density of $2 \times 10^{4} / \mathrm{ml}$ and treated with the indicated concentrations of naringenin for $24 \mathrm{~h}$. Cells were then incubated with fresh medium containing $5.0 \mathrm{~g} / 1 \mathrm{MTT}$ at $37^{\circ} \mathrm{C}$ for an additional $3 \mathrm{~h}$. Subsequent to washing with phosphate-buffered saline (PBS), the purple-blue sediments were dissolved in $1 \mathrm{ml}$ isopropanol and the absorbance was read at $563 \mathrm{~nm}$. The relative proliferation rate was calculated based on the optical density of each sediment at $563 \mathrm{~nm}$ compared with the vehicle-treated groups.

Western blot analysis. Cell lysates were obtained from the naringenin-treated TSGH-8301 cells. A total of $50 \mu \mathrm{g}$ protein was separated using $10 \%$ polyacrylamide gels and electrotransferred to nitrocellulose membranes. Membranes were blocked using PBS containing $0.5 \%$ non-fat milk for $1 \mathrm{~h}$ at room temperature and then probed with anti-MMP2 (Millipore, Billerica, MA, USA), anti-NFKB (BD Biosciences, Franklin Lakes, NJ, USA), anti-AKT, anti-phospho-AKT, anti-ERK, anti-p38, and-phospho-p38 and anti- $\beta$-actin (Santa Cruz Biotechnology Inc., Santa Cruz, CA, USA) primary antibodies at room temperature for $1 \mathrm{~h}$. The membranes were subsequently washed with PBS containing $0.1 \%$ Tween-20 and incubated with horseradish peroxidase-conjugated bovine anti-goat immunoglobulin $\mathrm{G}$ antibodies at a dilution of 1:5,000 (Santa Cruz Biotechnology, Inc.). Membranes were washed with PBS, prior to detection of the reactive signal using enhanced chemiluminescence (Amersham Pharmacia Biotech, Amersham, UK). $\beta$-actin expression was used as an internal control.

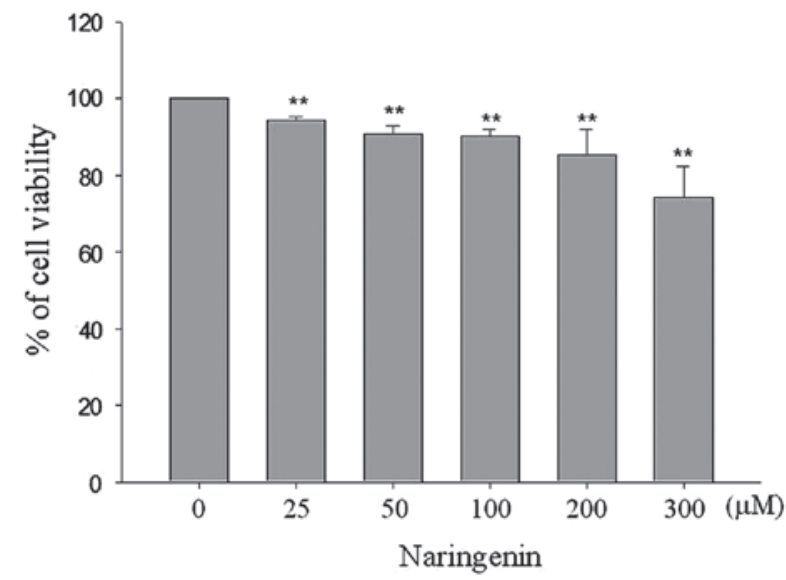

Figure 1. Effect of naringenin on the viability of TSGH-8301 bladder cancer cells. TSGH-8301 cells were treated with the indicated concentrations of naringenin for $24 \mathrm{~h}$ and cell viability was measured using the MTT assay. Data are presented as the mean \pm standard deviation from a minimum of three independent experiments. ${ }^{* *} \mathrm{P}<0.01$ compared with the control group.

Wound healing assay. For the wound healing experiments, cells were seeded at a density of $1 \times 10^{6} / \mathrm{ml}$. A wound was created by scraping the cell monolayer with a sterilized tip. Following the removal of the detached cells, the cell monolayer was treated with the indicated concentrations of naringenin for $24 \mathrm{~h}$. Migration was assessed by counting the cell numbers in the cell-free region. The value was presented as the mean \pm standard deviation (SD) of three independent experiments.

Gelatin zymography assay. Cells were incubated in serum-free medium and cultured in the presence of the indicated naringenin concentrations for $24 \mathrm{~h}$. Supernatants were then collected and separated using $8 \%$ SDS-polyacrylamide gels containing $0.1 \%$ gelatin. The gels were washed with washing buffer $(2.5 \%$ Triton X-100) twice with gentle agitation at room temperature for $30 \mathrm{~min}$, prior to incubation with the reaction buffer $\left[40 \mathrm{mM}\right.$ Tris- $\mathrm{HCl}(\mathrm{pH} 8.0) 10 \mathrm{mM} \mathrm{CaCl}_{2}$ and $0.01 \% \mathrm{NaN}_{3}$ ] for $12 \mathrm{~h}$. The gels were then stained with Coomassie Brilliant Blue R250 and destained. The degraded zones, representing MMP-2 activity, were quantified using a densitometer.

Migration assay. Cells were treated with different concentrations of naringenin for $24 \mathrm{~h}$ and seeded at a density of $5 \times 10^{5 / \mathrm{ml}}$ in the upper chamber of the 48-well Boyden chamber. The lower chamber contained 20\% FBS. The chamber was incubated at $37^{\circ} \mathrm{C}$ for $24 \mathrm{~h}$. Cells that migrated to the lower surface of the membrane were fixed in methanol for $10 \mathrm{~min}$ and stained with $10 \%$ Giemsa for $1 \mathrm{~h}$. The number of cells was quantified by counting three random microscopic fields (magnification, $\mathrm{x} 400$ ).

Statistical analysis. Data are presented as the mean \pm SD of three independent experiments and were evaluated using the Student's t-test with SPSS statistical software (SPSS, Inc., Chicago, IL, USA). A value of $\mathrm{P}<0.05$ was considered to indicate a statistically significant difference. 
A

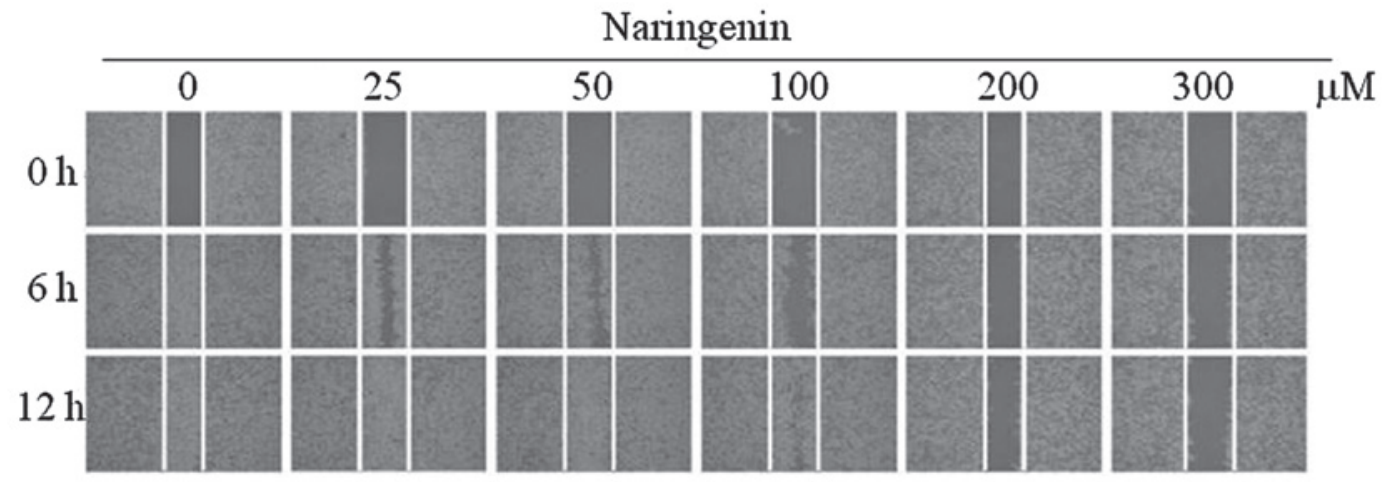

B Naringenin

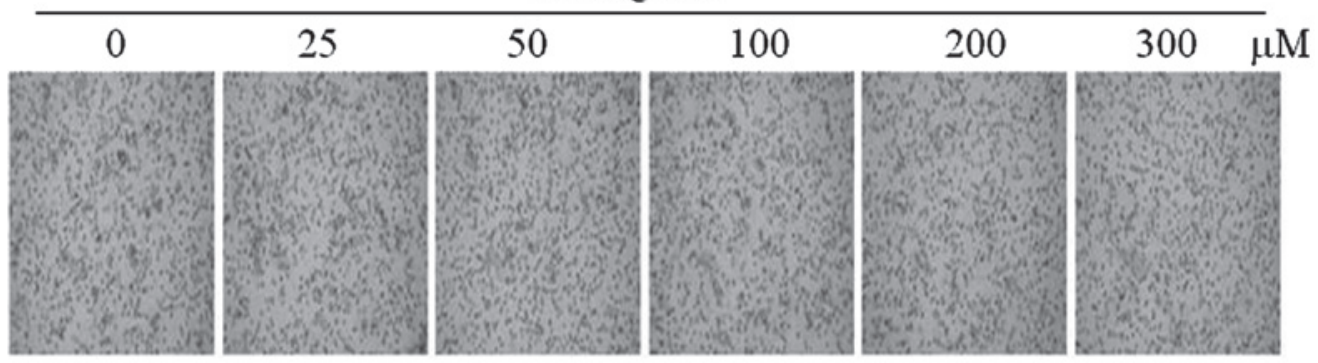

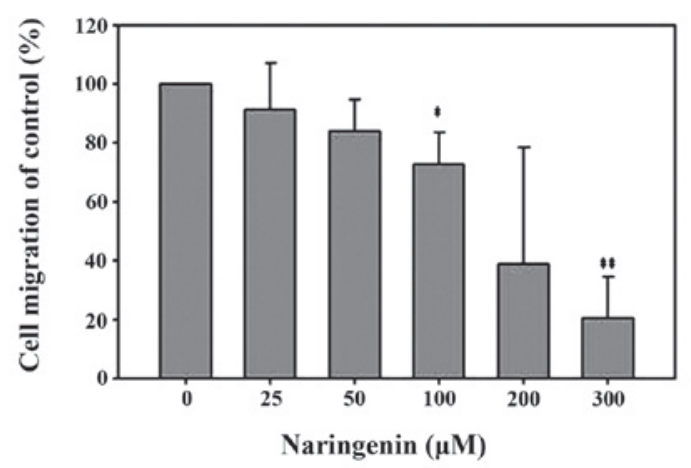

Figure 2. Naringenin inhibits TSGH-8301 cell migration in a dose-dependent manner. (A) In the wound healing assay, a monolayer of $80 \%$ confluent TSGH- 8310 cells was scratched using a pipette tip. Cells were then treated with the indicated concentrations of naringenin for 6 and $12 \mathrm{~h}$. Images were captured using a phase contrast microscope (magnification, x100). (B) In the migration assay, cells were treated with varying concentrations of naringenin. The migrated cells were then stained with Giemsa and counted. Data are presented as the mean \pm standard deviation from a minimum of three independent experiments, with the percentage of cell migration in the control set to $100 \%$. ${ }^{*} \mathrm{P}<0.05$ and ${ }^{* *} \mathrm{P}<0.01$ compared with the control group.

\section{Results}

Naringenin reduces TSGH-8301 cell viability. In order to determine the effect of naringenin on the proliferation of bladder cancer cells, an MTT assay was conducted. As shown in Fig. 1, exposure to 25, 50, 100, 200 and $300 \mu \mathrm{M}$ naringenin for $24 \mathrm{~h}$ was observed to reduce cell viability to $94.38 \pm 0.93$, $90.59 \pm 2.29,89.92 \pm 1.98,85.34 \pm 6.63$ and $74.09 \pm 8.27 \%$, respectively, compared with the non-treated groups. To further assess the effect of naringenin on bladder cancer cell cycle distribution, flow cytometric analysis was performed. Naringenin treatment was not found to affect cell cycle distribution in TSGH-8301 cells (data not shown).

Naringenin reduces TSGH-8901 cell migration. In order to investigate the effect of naringenin on the migration of bladder cancer cells, wound healing and Boyden chamber assays were performed. Exposure to 100, 200 and $300 \mu \mathrm{M}$ naringenin for $12 \mathrm{~h}$ was observed to significantly decrease the motility of TSGH-8301 cells, consequently reducing the number of cells in the wound zone, compared with vehicle-treated control groups (Fig. 2A). Boyden chamber analysis indicated that migration was decreased to $91.40 \pm 15.78,84.10 \pm 10.75,72.75 \pm 10.76$, $39.00 \pm 39.44$ and $20.74 \pm 13.93 \%$ in cells treated with 25,50 , 100, 200 and $300 \mu \mathrm{M}$ naringenin, respectively (Fig. 2B).

Naringenin inhibits the expression and activity of migration-related proteins. It is well established that MMP-2 and urokinase-type plasminogen activator play an important role in cancer migration. To investigate whether naringenin modulates the expression and activity of MMP-2, western blot analysis and zymography assays were conducted. Naringenin 
A
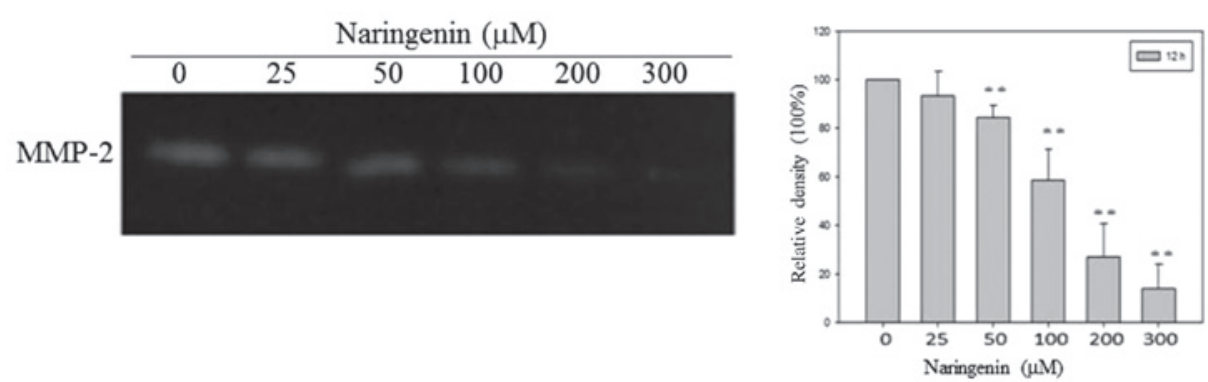

B

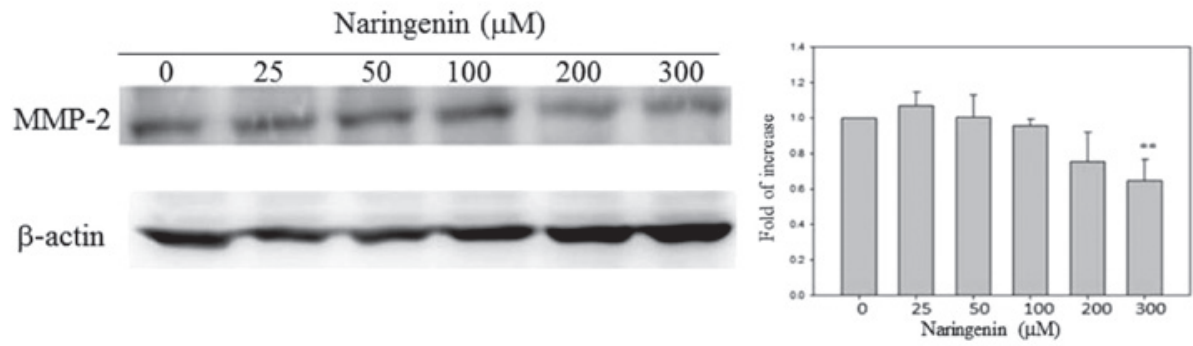

Figure 3. Effect of naringenin on the activity and expression of MMP-2 in TSGH-8301 cells. (A) TSGH- 8301 cells were treated with the indicated concentrations of naringenin for $24 \mathrm{~h}$. Conditional media were collected and MMP-2 activity was determined using gelatin zymography analysis. MMP-2 activity was quantified using a densitometer. (B) Equal quantities of cell lysates, which were extracted from cells that were treated with the indicated concentrations of naringenin for $24 \mathrm{~h}$, were subjected to western blot analysis using the anti-MMP-2 antibody. $\beta$-actin was used as an internal control. Densitometric data are presented as the mean \pm standard deviation compared with the control group, from a minimum of three independent experiments. ${ }^{* *} \mathrm{P}<0.01$ compared with the control group. MMP, matrix metalloproteinase.

A

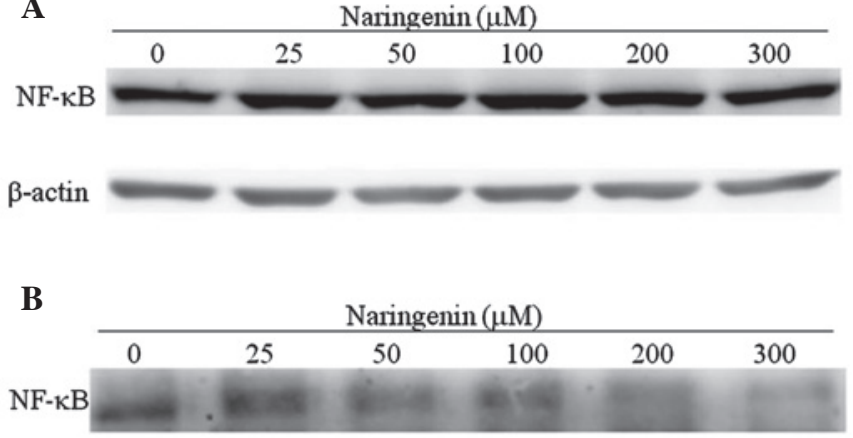

C-23

Figure 4. Naringenin inhibits NF- $\mathrm{kB}$ nuclear translocation. (A) Total cell lysates and (B) nuclear extracts obtained from cells treated with the indicated concentrations of naringenin for $24 \mathrm{~h}$ were subjected to western blot analysis using anti-NF- $\mathrm{KB}$ antibodies. $\beta$-actin and $\mathrm{C}-23$ were used as internal controls. NF- $\mathrm{kB}$, nuclear factor $\kappa$-light-chain-enhancer of activated B cells.

was found to inhibit MMP-2 activity in a dose-dependent manner, as revealed by zymography assay (Fig. 3A). However, a statistically significant decrease in MMP-2 expression was only observed in the TSGH-8301 cells treated with a high concentration of naringenin $(300 \mu \mathrm{M})$ (Fig. 3B).

The NF- $\mathrm{KB}$ transcription factor regulates numerous genes involved in proliferation, apoptosis and migration upon translocation to the nucleus (12). In the present study, western blot analysis was performed using whole cell or nuclear extracts to assess the effect of naringenin on NF- $\mathrm{KB}$ expression and nuclear translocation. As shown in Fig. 4A, naringenin treatment was not found to significantly alter NF- $\mathrm{\kappa B}$ expression. However, naringenin treatment was observed to significantly
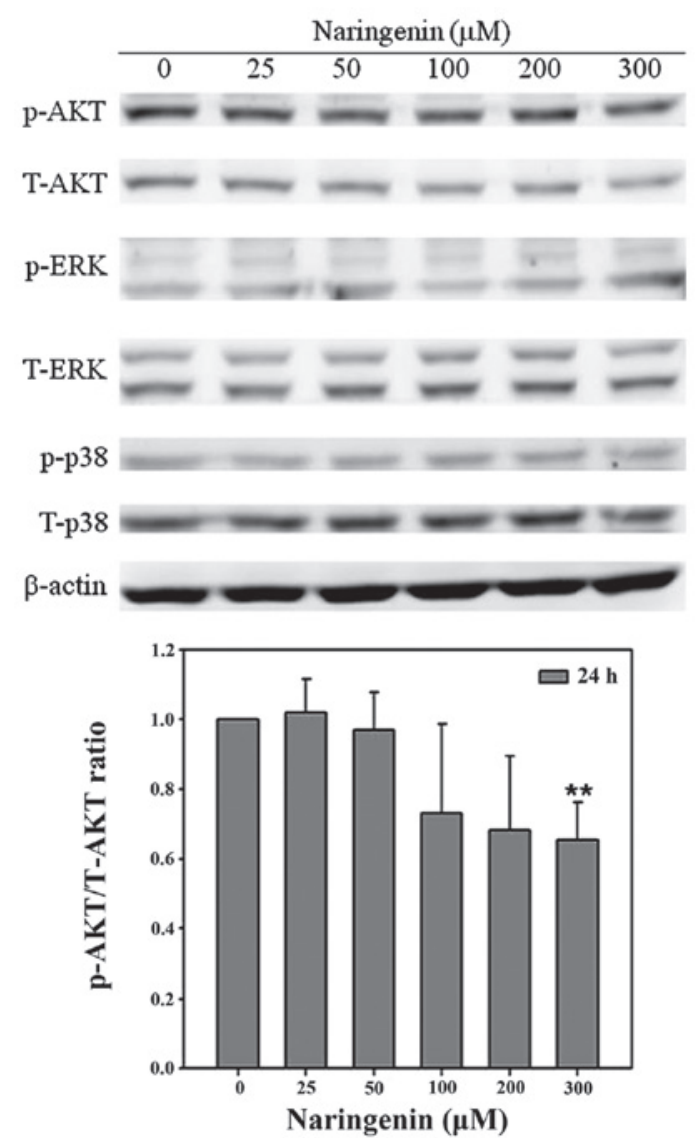

Figure 5. Naringenin attenuates AKT activity. Total cell lysates from cells treated with the indicated concentrations of naringenin for $24 \mathrm{~h}$ were subjected to western blot analysis using the indicated antibodies. $\beta$-actin was used as an internal control. Densitometric data are presented as the mean \pm standard deviation compared with the control group, from a minimum of three independent experiments. ${ }^{* *} \mathrm{P}<0.01$ compared with the control group. ERK, extracellular signal-regulated kinase; p-, phosphorylated; T-, total. 
decrease the nuclear translocation of $N F-\kappa B$ in a dose-dependent manner (Fig. 4B).

AKT and mitogen-activated protein kinase (MAPK) signaling, occurring in response to extracellular stimuli, have an important role in cell proliferation and migration. In order to investigate the effect of naringenin on AKT and MAPK activity, western blot analysis was performed. In the presence of 25, 50, 100, 200, $300 \mathrm{mM}$ naringenin, the $\mathrm{p}-\mathrm{AKT} / \mathrm{AKT}$ ratio was $0.94,0.90,1.16,0.69$ and 0.66 compared with the vehicle group. Naringenin was observed to significantly decrease AKT activity, as shown by the decreased p-AKT/T-AKT ratio in Fig. 5. However, naringenin was not identified to significantly alter the activity of p38 or ERK (Fig. 5).

\section{Discussion}

Bladder cancer is one of the primary urothelial malignancies, spreading into proximal or distal tissues through migration and invasion into the blood or lymphatic systems and causing high mortality rates. The suppression of migration may enhance the survival rate of patients with bladder cancer. In the present study, naringenin treatment was found to significantly repress the migration of bladder cancer cells through the downregulation of MMP-2 and the inhibition of AKT and $\mathrm{NF}-\kappa \mathrm{B}$ activities.

It is well established that degradation of the extracellular matrix (ECM) causes metastasis by enabling the spread of cancer cells through blood and lymphatic vessels (13). Levels of MMPs, a family of zinc-dependent ECM endopeptidases, have been shown to be significantly elevated in metastatic human cancers (14). Citrus flavonoids suppress cancer cell migration through the downregulation of MMP expression. Nobiletin, a major bioactive citrus flavonoid, is capable of reducing the MMP-7 proenzyme (proMMP-7) expression by inhibiting the binding of activator protein-1 to the proMMP-7 promoter in colorectal cancer cells (15). In addition, nobiletin exhibits anti-metastatic effects by modulating chemokine receptor-4 and MMP-9 expression in breast cancer cells (16). Consistent with these observations, the present study revealed that naringenin reduced MMP-2 expression and activity, which attenuated the migration of bladder cancer cells. Furthermore, treatment with naringenin significantly reduced the nuclear translocation of $\mathrm{NF}-\kappa \mathrm{B}$. The NF- $\kappa \mathrm{B}$ transcription factor regulates numerous cellular functions, including adhesion, inflammation and cancer metastasis, by controlling the expression of several genes, such as cyclooxygenase-2 (COX-2), MMP-2 and MMP-9 (17). In the normal state, NF- $\kappa \mathrm{B}$ is bound to the cytoplasmic region, but is translocated into the nucleus in response to certain stimuli (17). Inhibiting the function of natural bioactive compounds may be a promising chemotherapeutic strategy (17). A recent study demonstrated that naringenin administration downregulated NDEA-induced NF- $\kappa$ B, COX-2, MMP-2 and MMP-9 expression and inhibited NDEA-induced hepatocellular carcinomas in rats (8). In the present study, naringenin was found to reduce $\mathrm{NF}-\kappa \mathrm{B}$ nuclear translocation and inhibit MMP-2 expression, subsequently decreasing bladder cancer cell migration.

Phosphorylation-induced AKT activation leads to the phosphorylation of downstream targets, including glycogen synthase kinase- $3 \beta$, forkhead transcription factors and $N F-\kappa B$, which in turn mediate the effects of AKT on cell proliferation, apoptosis and migration (18). AKT phosphorylation has been found in several human cancers and correlates with poor prognosis (19). Nobiletin has been shown to attenuate the migration and invasion of gastric adenocarcinomas by downregulating AKT signals (20). Similarly, fisetin exhibits antimetastatic effects by deactivating AKT and reducing MMP-23 expression in PC-3 prostate cancer cells (21). The present study revealed that naringenin inhibited AKT phosphorylation, which suggests that the targeting of AKT phosphorylation may have a role in the anticancer effects of naringenin.

In conclusion, the present study has provided the first evidence, to the best of our knowledge, that naringenin is a novel MMP-2 inhibitor that inhibits bladder cancer cell migration and, thus, may have the potential to suppress bladder cancer metastasis.

\section{Acknowledgements}

This study was supported by grants from the National Science Council of Taiwan (nos. NSC-101-2311-B-040-001 and CSMU-CMMC-102-01).

\section{References}

1. Tian B, Wang Z, Zhao Y, et al: Effects of curcumin on bladder cancer cells and development of urothelial tumors in a rat bladder carcinogenesis model. Cancer Lett 264: 299-308, 2008.

2. Sun F, Zheng XY, Ye J, Wu TT, Wang JI and Chen W: Potential anticancer activity of myricetin in human T24 bladder cancer cells both in vitro and in vivo. Nutr Cancer 64: 599-606, 2012.

3. Zhang L, Chen W and Li X: A novel anticancer effect of butein: inhibition of invasion through the ERK1/2 and NF-kappaB signaling pathways in bladder cancer cells. FEBS Lett 582: 1821-1828, 2008.

4. Chen NG, Lu CC, Lin YH, et al: Proteomic approaches to study epigallocatechin gallate-provoked apoptosis of TSGH-8301 human urinary bladder carcinoma cells: roles of AKT and heat shock protein 27 -modulated intrinsic apoptotic pathways. Oncol Rep 26: 939-947, 2011.

5. Qin J, Wang Y, Bai Y, et al: Epigallocatechin-3-gallate inhibits bladder cancer cell invasion via suppression of NF- $\mathrm{B}$ mediated matrix metalloproteinase-9 expression. Mol Med Rep 6: 1040-1044, 2012.

6. Frydoonfar HR, McGrath DR and Spigelman AD: The variable effect on proliferation of a colon cancer cell line by the citrus fruit flavonoid Naringenin. Colorectal Dis 5: 149-152, 2003.

7. Lentini A, Forni C, Provenzano B and Beninati S: Enhancement of transglutaminase activity and polyamine depletion in B16-F10 melanoma cells by flavonoids naringenin and hesperitin correlate to reduction of the in vivo metastatic potential. Amino Acids 32: 95-100, 2007.

8. Subramanian P and Arul D: Attenuation of NDEA-induced hepatocarcinogenesis by naringenin in rats. Cell Biochem Funct 31: 511-517, 2013

9. Chen S, Ding Y, Tao W, Zhang W, Liang T and Liu C: Naringenin inhibits TNF- $\alpha$ induced VSMC proliferation and migration via induction of HO-1. Food Chem Toxicol 50: 3025-3031, 2012.

10. Lou C, Zhang F, Yang M, et al: Naringenin decreases invasiveness and metastasis by inhibiting TGF- $\beta$-induced epithelial to mesenchymal transition in pancreatic cancer cells. PLoS One 7: e50956, 2012.

11. Kim JJ: Recent advances in treatment of advanced urothelial carcinoma. Curr Urol Rep 13: 147-152, 2012.

12. Min C, Eddy SF, Sherr DH and Sonenshein GE: NF-kappaB and epithelial to mesenchymal transition of cancer. J Cell Biochem 104: 733-744, 2008.

13. Klein T and Bischoff R: Physiology and pathophysiology of matrix metalloproteases. Amino Acids 41: 271-290, 2011.

14. Gialeli C, Theocharis AD and Karamanos NK: Roles of matrix metalloproteinases in cancer progression and their pharmacological targeting. FEBS J 278: 16-27, 2011. 
15. Kawabata K, Murakami A and Ohigashi H: Nobiletin, a citrus flavonoid, down-regulates matrix metalloproteinase-7 (matrilysin) expression in HT-29 human colorectal cancer cells. Biosci Biotechnol Biochem 69: 307-314, 2005.

16. Baek SH, Kim SM, Nam D, et al: Antimetastatic effect of nobiletin through the down-regulation of CXC chemokine receptor type 4 and matrix metallopeptidase-9. Pharm Biol 50: 1210-1218, 2012.

17. Luqman S and Pezzuto JM: NFkappaB: a promising target for natural products in cancer chemoprevention. Phytother Res 24: 949-963, 2010

18. Sheng S, Qiao M and Pardee AB: Metastasis and AKT activation. J Cell Physiol 218: 451-454, 2009.
19. Cicenas J: The potential role of Akt phosphorylation in human cancers. Int J Biol Markers 23: 1-9, 2008.

20. Lee YC, Cheng TH, Lee JS, et al: Nobiletin, a citrus flavonoid, suppresses invasion and migration involving FAK/PI3K/Akt and small GTPase signals in human gastric adenocarcinoma AGS cells. Mol Cell Biochem 347: 103-115, 2011.

21. Chien CS, Shen KH, Huang JS, Ko SC and Shih YW: Antimetastatic potential of fisetin involves inactivation of the PI3K/Akt and JNK signaling pathways with downregulation of MMP-2/9 expressions in prostate cancer PC-3 cells. Mol Cell Biochem 333: 169-180, 2010. 